\title{
MAMMARY FISTULA - FROM CONGEPT TO REALITY
}

\section{Iordache Ionut-Eduard 1,2, Unc Octavian ${ }^{1,2}$, Costea Dan ${ }^{1,2}$, Popescu Razvan ${ }^{1,2}$, Neacsu Sabina ${ }^{1,2}$, Grama Mirela1,2, Steriu Liliana ${ }^{1,2}$, Leopa Nicoleta ${ }^{1,2}$, Baltatescu Gabriela, ${ }^{1,2}$, Tomulescu Maria ${ }^{1,2}$}

${ }^{1}$ Faculty of Medicine, University "Ovidius" of Constanta

${ }^{2}$ First Department of General Surgery, Emergency County Hospital Constanta

\begin{abstract}
Breast fistula belongs to the group of chronic mammary suppuration, one pathology marked by several reappearance, apparently without a cause, which raise many problems both for the patient and surgeon. One characteristic of this pathology is the microbial mammary abcess which untreated develops into fistula.

The possibility of uninfectious mastitis less specified it's still the most usual reason for mastitis. This thing rise questions regarding clinics,pathology and treatment.

This were relyes on a retrospective and prospective study of a number of 55 cases represented by patients with mammary infections which were diagnosed and surgicaly treated in Surgery Clinic of the Constanta County Hospital between 2014-2018.

Mammary fistulas that appear as a consequence of amicrobial breast abscesses are characterized by multiple recurrences, even if they are treated by surgery through excision of the fistulous path.

Numerous studies are trying to elucidate the cause of this pathology. There are many hypotheses, but from the studies we have found that smoking is an important etiological factor, having toxic effect through double mechanism :

- directly - on the retroareolar lactiferous duct.

- indirectly - hormonally, by stimulating lacteous secretion.

Both hypotheses explain why this condition is recurrent.

Treatment of mammary fistulas requires both smoking cessation and proper surgical treatment. Studies have shown that a simple excision of the fistula tract is not sufficient and leads to recurrences. A wide, sectoral intervention must be practiced, with excision taking the ductal branches that underlie the recurrences.
\end{abstract}

Keywords : mammary fistula, smoking, wide excision.

\section{Introduction}

Mammary fistula is an abnormal communication of the mammary ducts with the skin, being included in the group of chronic breast suppurations, a pathology characterized by numerous recurrences, apparently without cause, which raises problems for both the patient and the surgeon. A feature of this pathology is the amicrobial mammary abscess that untreated evolves into fistulization (1).

This condition does not appear exclusively in women. Mammary fistulas that appear in men are very rare, the first being described in 1974 by Tedeschi and colleagues. Research into the literature revealed 20 cases of breast fistula in men, a number that includes 3 cases in children and 3 cases associated with HIV infection.

Lactation abscesses are less complicated with fistulization because in the initial stages they respond to antibiotic treatment and the surgical treatment leads to healing (2).

Non-infectious mastitis occurs outside pregnancy and has a capricious evolution until the appearance of amicrobial abscess.

The differential diagnosis should be made with carcinomatous mastitis, which is not always easy.

The diagnostic certainty is histological and cytological. 
The evolution of these mastitis is capricious, with recurrences, variable from one woman to another, apparently without cause.

Clinically, there is an tumefaction of the periareolar inflammation, which retracts the mamelon, with redness, heat, pain, edema of the skin. Broad-spectrum antibiotics are ineffective. The consequence is the formation of an amicrobial abscess, a lesion that can spontaneously fistulize at the tegument. After the surgical treatment the fistula is cleansed, the patient seems to be cured for several weeks or months, then a recurrence of the abscess follows, with an identical clinical picture and fistulisation in the same place (3).

The location of the fistula is characteristic, periareolar or in the mamelono-areolar duct.

\section{Material and Method}

This research is based on a retrospective and prospective study of 55 cases of patients with mammary infections, diagnosed and surgically treated at the Surgery Clinic of the Constanta County Emergency Hospital, between 2014-
2018. The study aimed to highlight the elements related to the etiology and pathology of mammary fistulas and the statistical interpretation of the data, although the batch is only 55 patients, we searched on the formulas used to take into account the representative value of the numeric or percentage value data we obtained.

\section{Results}

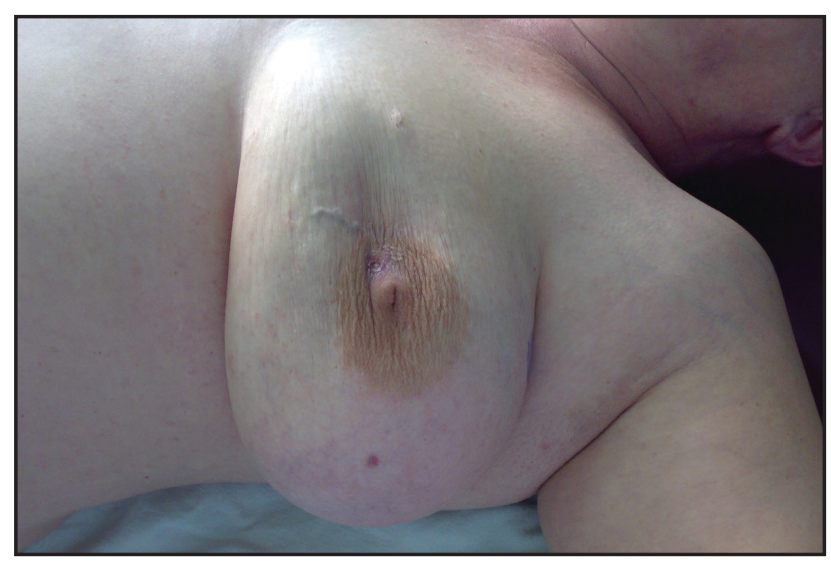

Fig. no. 1 - Mammary Fistula.

Table no. 1: Frequency and distribution of cases of mammary pathology by calendar years.

\begin{tabular}{|l|c|c|c|c|c|c|}
\hline Year & 2014 & 2015 & 2016 & 2017 & 2018 & Total \\
\hline Number of admissions & 5728 & 3704 & 4454 & 3660 & 3460 & 21006 \\
\hline Number of operations & 3978 & 3539 & 4279 & 2856 & 2946 & 17598 \\
\hline Number of cases of breast pathology & 170 & 224 & 126 & 209 & 211 & 940 \\
\hline $\begin{array}{l}\text { Number of cases of malignant breast } \\
\text { pathology }\end{array}$ & 84 & 108 & 59 & 64 & 87 & 402 \\
\hline $\begin{array}{l}\text { Number of cases of benign breast } \\
\text { pathology }\end{array}$ & 86 & 116 & 67 & 145 & 124 & 538 \\
\hline $\begin{array}{l}\text { Number of cases of breast infections } \\
\text { infections } \% \text { of the total number of cases } \\
\text { of benign pathology (tumors) }\end{array}$ & $3.48 \%$ & $6.03 \%$ & $17.91 \%$ & $10.34 \%$ & $14.51 \%$ & $10.22 \%$ \\
\hline
\end{tabular}

Table no. 2: Frequency and distribution by year of the types of mammary infections.

\begin{tabular}{|l|c|c|c|c|c|c|}
\hline Year & 2014 & 2015 & 2016 & 2017 & 2018 & Total \\
\hline Mammary Fistulas & 1 & 2 & 8 & 10 & 11 & $32(58.18 \%)$ \\
\hline$\%$ & $3.12 \%$ & $6.25 \%$ & $25 \%$ & $31.25 \%$ & $34.37 \%$ & $100 \%$ \\
\hline $\begin{array}{l}\text { Mammary } \\
\text { abscesses }\end{array}$ & 1 & 4 & 2 & 3 & 6 & $16(29.09 \%)$ \\
\hline$\%$ & $6.25 \%$ & $25 \%$ & $12.5 \%$ & $18.75 \%$ & $37.5 \%$ & $100 \%$ \\
\hline Acute mastitis & 1 & 1 & 2 & 1 & 1 & $6(10.9 \%)$ \\
\hline$\%$ & $16,66 \%$ & $16.66 \%$ & $33.33 \%$ & $16.66 \%$ & $16.66 \%$ & $100 \%$ \\
\hline TB mammary & 0 & 0 & 0 & 1 & 0 & $1(1.81 \%)$ \\
\hline$\%$ & $0 \%$ & $0 \%$ & $0 \%$ & $100 \%$ & $0 \%$ & $100 \%$ \\
\hline Total & 3 & 7 & 12 & 15 & 18 & $55(100 \%)$ \\
\hline$\%$ & $5.45 \%$ & $12.72 \%$ & $21.81 \%$ & $27.27 \%$ & $32.72 \%$ & $100 \%$ \\
\hline
\end{tabular}




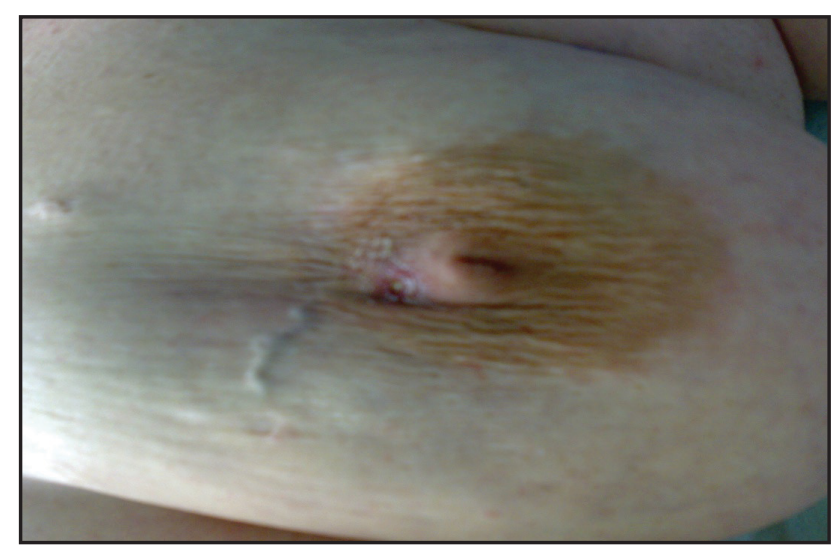

Fig. no. 2 - Mammary Fistula.

Table no. 3: Age distribution of mammary fistulas.

\begin{tabular}{|l|c|c|}
\hline Age group & $\begin{array}{l}\text { Number of } \\
\text { mammary fistula } \\
\text { cases }\end{array}$ & \\
\hline$<20$ years & 2 & $6.25 \%$ \\
\hline $20-29$ years & 4 & $12.50 \%$ \\
\hline $30-39$ years & 10 & $31.25 \%$ \\
\hline $40-49$ years & 12 & $37.50 \%$ \\
\hline $50-59$ years & 4 & $12.50 \%$ \\
\hline $60-69$ years & 0 & $0 \%$ \\
\hline$>70$ years & 0 & $0 \%$ \\
\hline Total & 32 & $100 \%$ \\
\hline
\end{tabular}

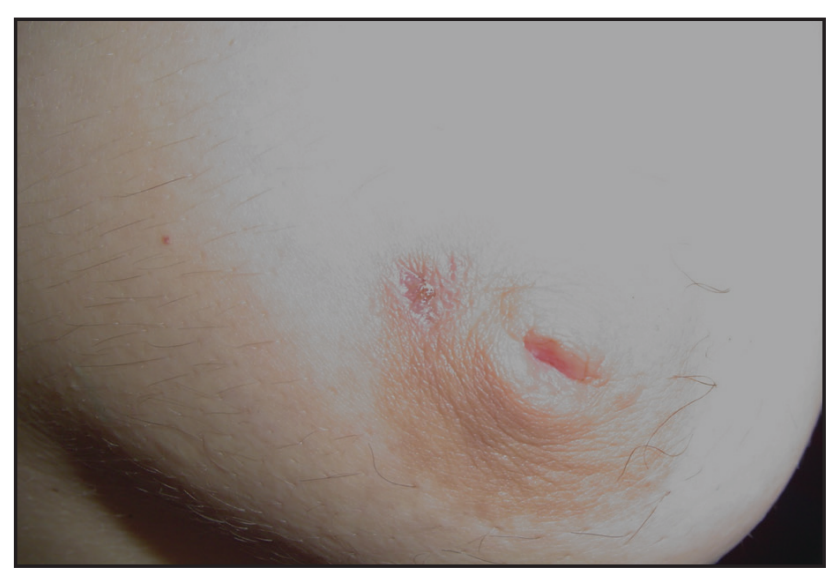

Fig. no. 3 - Mammary Fistula.

Table no. 4: Types of surgery for mammary fistulas.

\begin{tabular}{|l|c|c|}
\hline Operation type & $\begin{array}{c}\text { Number of mammary } \\
\text { fistula cases }\end{array}$ & $\%$ \\
\hline $\begin{array}{l}\text { Excision of the fistulous } \\
\text { path }\end{array}$ & 47 & $85.45 \%$ \\
\hline Breast sectorectomy & 4 & $7.27 \%$ \\
\hline $\begin{array}{l}\text { Lumpectomy with } \\
\text { removal of the fistula and } \\
\text { partially the mamelon }\end{array}$ & 4 & $7.27 \%$ \\
\hline Total & 55 & $100 \%$ \\
\hline
\end{tabular}

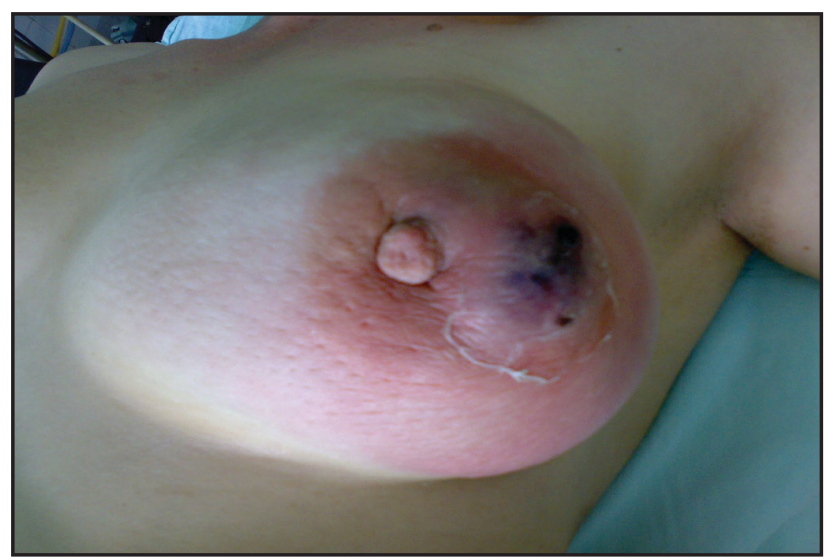

Fig. no. 4 - Mammary fistula - intraoperative appearance

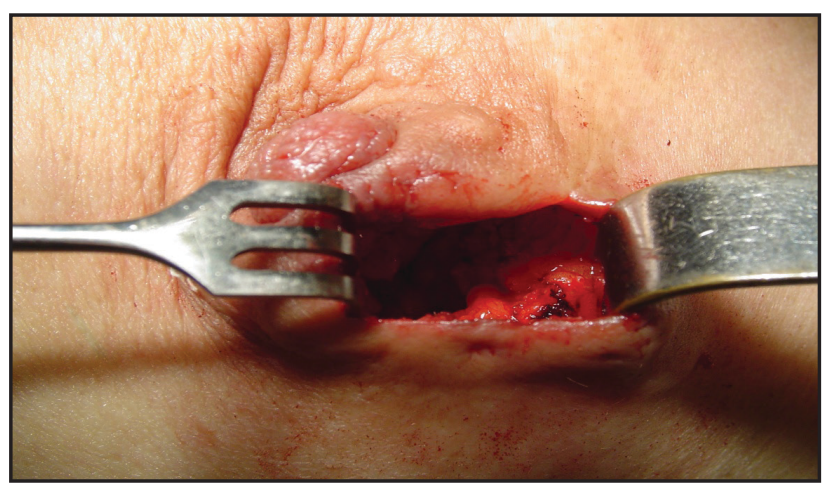

Fig. no. 5 - Mammary fistula - intraoperative appearance

\section{Discussion}

\section{Defining the diagnosis of mastitis and}

\section{semantic differentiation}

In senological surgical pathology, several terms are used that define the various acute or chronic inflammatory phenomena, specific or nonspecific of mammary glandular tissue. The localization of mammary suppurative phenomena in the preglandular, retroglandular or mammary gland tissue is a classic classification, with well-defined notions. In the study we did, we used four terms that we tried to make different semantic meaning to differentiate the studied breast pathology. Thus the notion of mammary infection was equated semantically with that of mastitis, in general understanding by this way all the inflammatory phenomena of mammary, indifferent of etiology, pathophysiology or clinical form (4).

In the subsequent presentation of the results we differentiated within this notion of mastitis 
four clinical entities, respectively:

- mammary abscesses, with determinism and identification of the pathogenic microbial agent, this category including all nonspecific mammary suppurations, except lactation mastitis.

- lactation mastitis, in which the development of suppurative phenomena occurred within the perimeter of the lactation period, the lactation-gate correlation of microbial entry being evident.

- a unique case in the whole case study during the five years studied and summing a total of over 1000 cases, constituted the detection of a case of tuberculous mastitis, with the identification of the specific microbial agent, the Koch bacillus.

- a fourth category is practically the group of patients with inflammatory diseases diagnosed as mammary fistulas, terminology that we borrowed from the literature.

This moment actually constituted the introduction for the first time in the current Romanian surgical context of the notion of mammary fistula, the first terminological mention being made within the Romanian Surgery Society, the Constanta branch, in November 2004.

We mention that out of the group of 55 patients only 32 were classified, due to their clinical and etiological characteristics, as mammary fistulas, a conspicuously insufficient number for a representative statistical study, but on the basis of which we performed the present work.

Thus, we were obliged to summarize only the cases identified by us in the Surgery Clinic of the Constanta County Emergency Clinical Hospital, without including a number of other approximately 16 cases of patients diagnosed with mammary fistulas (in the semantic context of the study), but in which only drug treatment was performed, with or without local topics, and in which the remission of inflammatory phenomena determined the patients not to present for surgery.

We excluded from our research group all the other forms of mastitis, the etiology of the appearance of these diseases being markedly different from what we tried to highlight as a pathogenic mechanism for the mammary fistula.

\section{The incidence of mammary fistulas}

in the surgical pathology within the Surgery Clinic of the Constanta County Emergency

\section{Clinical Hospital.}

Table 1 shows that surgically sanctioned mammary infections account for $5.85 \%$ of all cases diagnosed with a mammary condition.

The question that can be asked about this pathology (mammary fistulas) is the following: it is a disease without clinical interest given the rarity of the cases or, although the cases are met with an average value of about 6 cases in a year, it raises problems diagnosis and especially therapeutic?

We may also wonder if this condition is discovered at the actual values of its incidence among women or what happens in our Surgery Clinic is just the tip of an iceberg phenomenon. In this case, too, the question would be whether more effective diagnostics should be taken.

Another question could also be, starting from the incidence, whether the mammary fistulas, to the percentage values encountered, affects or not the quality of the patient's life, hence the practical importance of diagnosis and knowledge of this condition.

If we evaluate the types of inflammatory mammary diseases over the five years, according to the four large groups we established, we find that the infectious mammary pathology is dominated by the mammary fistula, with a percentage of $\sim 60 \%$, followed by microbial mammary abscesses, found in $\sim 30 \%$ and acute mastitis, with an incidence of $\sim 10 \%$.

These cases as surgical presentation and sanction probably depend strictly on the addressability of the patient.

\section{Distribution of mammary fistulas by} age groups.

From the tables no. 6 and no. 10 it is found that the highest percentages of a mammary fistula occur in the 3 and 4 decades of life, respectively 30-39 years and 40-49 years, which corresponds to the maximum activity age of the woman in all the points of view (physical, social, hormonal, etc.).

The question may arise why in patients over 60 years of age we have not encountered 
cases, especially if we analyze the phenomenon from the etiological point of view, because a smoker at 60-70 years categorically showed a greater aggression in comparison with the young patients .

We may wonder whether it is a concomitant hormonal component in the onset of this phenomenon of mammary fistula or whether there is a phenomenon of immunization or ,' vaccination,' in long-term smoking that no longer determines in this case mammary fistula.

We can also consider that a maximum incidence of smoking for young age is directly correlated with the histophysiological stability of the mammary gland, its changes related to the menstrual hormone cycle and the ,'youth ,' of the mammary gland.

\section{Diagnosis of mammary fistula}

The diagnosis of mammary fistula is based on two elements : the clinical examination and the anamnesis that highlight the smoker's history. For the studied group what marked the classification of patients in this category of mammary fistula was the anamnestic element of smokers, a factor of curiosity for the other doctors. Patients also look with lack of confidence when they are told that their condition has a direct correlation with smoking. The number of cigarettes smoked daily for our group is variable, with patients smoking 5-7 cigarettes/day but also patients with 2030 cigarettes/day, unable to make a correlation between the number of cigarettes smoked daily and the occurrence of the inflammatory condition. We would like to point out once again, that in total, patients hardly accept the idea that the „banal smoking” may have to do with mammary suppuration.

Another element is the differential diagnosis for all patients in the study group, seeking to eliminate lactation mastitis or specific mastitis, none of our patients having such a diagnosis. The easiest thing to do is to differentiate between lactation mastitis and mammary fistula where the absence of breastfeeding easily removes this diagnosis. The other condition with which the differential diagnosis is made involves the search for the pathological history or the current detection of some TB or fungal infections (5).

The differential diagnosis between classical mammary fistula and carcinomatous mastitis is relatively easy to perform and rarely necessary in practice, because while Volkmann mastitis is characterized by the presence of a quasi-total inflammatory breast, the mammary fistula presents as a periareolar fistulous orifice, a intermittent suppuration and rarely high-intensity inflammatory phenomena.

\section{Recurrence of mammary fistulas}

Mammary fistula, as a chronic suppuration, has a special, relapsing character, in the sense that the patient has no treatment, has variable periods of time, weeks or months of remission, in which the lesion is represented by a closed fistulous orifice, possibly covered by a crust or even by the disappearance of all inflammatory phenomena, followed by „re-awakening” or „resumption” of inflammation, with local, periareolar suppuration. A peculiarity of this inflammatory condition, encountered throughout the study, was the onset of the disease. In about $80 \%$ of the patients who first appeared in our clinic and were diagnosed with mammary fistula, the suppuration was important with certain mastitis phenomena, similar to identification with the manifestations of acute lactation mastitis, but with the difference that breastfeeding was absent (present or recent), instead being present the habit of smoking.

After the initial treatment, which is often represented by the incision and evacuation of the purulent collection, the evolution is favorable, with the mention that we observed in the studied group that the non-smoking cessation leads to the recurrent character of the condition. Patients appear for several months with the periareolar fistula orifice, with clinical and local elements of inflammatory activity. We found that patients who quit smoking have a favorable evolution with healing of the respective lesion. We had a patient in group who gave up smoking and whose subsequent evolution of the surgery was favorable, without recidivism episodes, but two years after the surgical treatment, in the conditions in which she started smoking again, the fistula relapsed. The patient refused the operation and could not be followed.

Paraclinical diagnosis in the mammary fistula is rarely performed, the clinical aspect determining both the patient and the doctor to 
intervene without further investigation.

However, a mammary ultrasound or mammography highlights the lesion without constituting a diagnosis of certainty.

The cytological examination constituted in the vast majority of cases from the microbial cultures of the purulent secretions does not bring relevant data, in the majority of cases a amicrobial culture is highlighted or in the other cases the presence of a staphylococcus.

If the surgical treatment is based on an excision of the fistulous path leading to a sectorectomy or quadrantectomy, the anatomopathological examination certifies the inflammatory ductal and periductal lesions (1).

\section{Smoking and mammary fistulas}

Patients with mammary fistulas that we studied during the five years were characterized by an evolution with numerous recurrences, despite the surgical treatment. We have tried to find an explanation for the recurrent nature of this pathology. From the anamnesis, it turned out that all patients were constantly smoking, some pacients even smoked more packs of cigarettes a day, so we wondered if it was a simple coincidence or in fact smoking really did play a much more important role in the pathology represented by the breast fistulas. Based on this idea, we researched the literature and discovered numerous studies that claim that smoking is an important etiological factor in the appearance and evolution of mammary fistulas.

Bundred NJ and co-workers performed an 11-year retrospective study on a batch of 122 mammary abscess patients and found that mammary fistulas developed more frequently in patients who were chronic smokers. The conclusion of this study was that smoking is important in the natural history of non-lactational abscess and may predispose to anaerobic breast infections and the development of breast fistulas (6).

After Salmon RJ smoking seems to be the only cause of recurrent breast abscesses outside the lactation period. The patients studied shared that they were chronic smokers (7).

A study by Schafer P. and colleagues in a group of 60 patients with recurrent subareolar mammary abscesses found a significant association with chronic cigarette smoking.
The strong association of cigarette smoking with recurrent subareolar mammary abscesses revealed a relative risk of 9.2 for a light smoker and 26.4 for a chronic smoker (8).

The conclusion of this study was that smoking would exert a direct toxic effect on the retroareolar lactiferous duct and indirectly on the hormonal pathway, with stimulation of the milk secretion, thus explaining the recurrent nature of this condition.

We discussed with patients with mammary fistulas who presented with recurrences and found that although they were informed that smoking cessation might stop the recurrence, the majority continued to smoke.

\section{Treatment of mammary fistulas}

Surgical treatment of mammary fistulas depending on the evolutionary moment at which the patient presents to the doctor has two variants: incision and evacuation of the purulent collection and mammary glandular resections, reduced or enlarged.

Mammary incision with evacuation of the abscess addresses the incipient stage of a mammary fistula when the patients present with a collection that includes all the celsian signs of inflammation. After the periareolar incision, the purulent collection is evacuated, followed by the local toilet with oxygenated water, betadine or other antiseptics.

The incision is the clinical element that ensures the momentary resolution. Normally patients should be recalled after an interval of about three months for excision of the remaining scar fistula pathway, but very few patients undergo this new intervention, the moment of return to the surgeon being determined by the activation of the fistula under continued smoking.

Breast glandular excisions may be reduced when the fistula pathway is extirpated, personally finding for the studied group that when the principle of extirpation of the glandular pyramid centered by the fistulous orifice was observed, the evolution was favorable.

Marking with a tracer represented by a dye (blue methylene) allows a good highlighting of the area to be removed, with the mention that in most cases it involved a large sectorectomy, with often unsightly results, especially for small 
breasts.

This removal is the only one that ensures healing of the fistula. The assertion made is valid only with the corollary of smoking cessation because in three of the patients in our group we have had relapses for years which puts into question the mechanism of the appearance of these fistulas, related to smoking, a mechanism that at least is not yet well defined (9).

Quitting smoking involves a psychological treatment that has only been performed by the surgeon.

The surgeon cannot argue for the patient's obligation to quit smoking except by other examples or by „waiting" for the patient to convince herself that smoking sustains the fistula. For about half of the patients in the group we considered that only the fear of a new surgery was the element that caused them to give up at least one period of smoking habit. After the study we have done we can say that there must be a collaboration between the surgeon and a psychologist or a mixed team will be formed to address these cases, the psychologist-surgeon pair leading to an increase in the chances of healing the mammary fistula.

Another surgery that has been proposed for mammary fistula is quadrantectomy or even subcutaneous mastectomy, techniques that have not been performed for our study except in one case. Mastectomy, however, is an exaggerated intervention with multiple psychological and aesthetic implications.

\section{Conclusions}

Mammary fistulae are a numerically reduced pathology in mammary disorders, but important due to the consequences of inadequate treatment, relapse and the paradoxical association of surgical treatment-smoking cessation, constituting the elements that create a certain psychosis for patients.

In the absence of smoking cessation it is doubtful that the mammary fistula will recur, which means beyond the surgical treatment the combination of the psychotherapeutic sequence to persuade patients to quit smoking.

The surgical treatment should not be reduced only to fistulectomy, but to wide extirpations, even with the risk of aesthetic deficiencies that can be corrected by plastic surgery techniques. Mastectomy in the case of breast fistulas seems to us an exaggerated gesture, which we did not find in the study group.

The paradox of the smoking-breast fistula association has determined us to look for the pathogenic explanation of the intervention of smoking in the production of the amicrobian abscesses of mammary fistula type, notions that in the specialized literature are little addressed at the moment, with practically the possibility of experimental studies on which we regretted that we could not do.

\section{General Conclusion}

Mammary fistulas are a reality of mammary pathology, the cases becoming more and more numerous, through a correct diagnosis and knowledge by the doctor of this association, smoking-fistula, which involves a double surgical and psychological approach for a therapeutic success.

The present paper is intended to signal this surgical clinical entity, MAMMARY FISTULA, for which the knowledge of both the surgeon and the patients the role of smoking in the etiology but especially in the therapeutic success (quitting smoking) may be beneficial.

\section{References}

1. Jatoi I, Kaufmann M, Petit JY. Atlas of breast surgery. 1 ed: Springer-Verlag Berlin Heidelber; 2006. 133 p.

2. Angelescu N. Tratat de patologie chirurgicala. Bucharest; 2003, pp. 1163-1169, 1176-1178

3. Schwartz S. Schwartz - Principiile chirurgiei. Bucharest: TEORA; 2005, pp. 544-549, 558560

4. Zollinger R. M. Zollinger Atlas de tehnici chirurgicale, Editura Ştiinţelor Medicale 2007, pp. 396-397

5. Gorins A. Aspects cliniques benigns du sein inflammatoire Journees Pyreneennes de Gynecologie - Tarbes 2\&3 octobre 1998

6. Bundred NJ, Dover MS, Coley S, Morrison 
JM. Breast abscesses and cigarette smoking. Br J Surg. 1992;79(1):58-9.

7. Salmon RJ. [Recurrent breast abscess: role of smoking]. J Gynecol Obstet Biol Reprod (Paris). 1996;25(3):242-3.

8. Schafer P, Furrer C, Mermillod B. An association of cigarette smoking with recurrent subareolar breast abscess. Int J Epidemiol. 1988;17(4):810-3.

9. Inflammations Breast Pathology, 2019 [Availablefrom:http://www.breastpathology. info/inflammations.html]. 